\title{
Indications for different types of brittle failure due to active coal mining using waveform similarities of induced seismic events
}

\author{
S. Wehling-Benatelli ${ }^{1}$, D. Becker ${ }^{2}$, M. Bischoff ${ }^{3, *}$, W. Friederich ${ }^{1}$, and T. Meier ${ }^{4}$ \\ ${ }^{1}$ Ruhr-University Bochum, Bochum, Germany \\ ${ }^{2}$ University of Hamburg, Hamburg, Germany \\ ${ }^{3}$ Federal Institute for Geosciences and Natural Resources (BGR), Hanover, Germany \\ ${ }^{4}$ Christian-Albrechts-University Kiel (CAU), Kiel, Germany \\ *now at: State Authority for Mining, Energy and Geology (LBEG), Hanover, Germany
}

Correspondence to: S. Wehling-Benatelli (sebastian.wehling@rub.de)

Received: 10 April 2013 - Published in Solid Earth Discuss.: 17 May 2013

Revised: 12 September 2013 - Accepted: 19 September 2013 - Published: 31 October 2013

\begin{abstract}
Longwall mining activity in the Ruhr coal mining district leads to mining-induced seismicity. For detailed studies the seismicity of a single longwall panel beneath the town of Hamm-Herringen in the eastern Ruhr area was monitored between June 2006 and July 2007 with a dense temporary network of 15 seismic stations. More than 7000 seismic events with magnitudes between $-1.7 \leq M_{\mathrm{L}} \leq 2.0$ were detected and localized in this period. Most of the events occurred in the vicinity of the moving longwall face.

In order to find possible differences in the brittle failure types of these events an association of the events to distinct clusters is performed based on their waveform characteristics. This task is carried out using a new clustering algorithm utilizing a network similarity matrix which is created by combining all available 3-component single station similarity matrices. The resultant network matrix is then sorted with respect to the similarity of its rows leading to a sorted matrix immediately indicating the clustering of the event catalogue. Finally, clusters of similar events are extracted by visual inspection.

This approach results in the identification of several large clusters which are distinct with respect to their spatial and temporal characteristics as well as their frequency magnitude distributions. Comparable clusters are also found with a conventional single linkage approach, however, the new routine seems to be able to associate more events to specific clusters without merging the clusters.
\end{abstract}

The nine largest observed clusters can be tentatively divided into three different groups that indicate different types of brittle failure. The first group consists of the two largest clusters which constitute more than half of all recorded events. Results of a relative relocation using cross-correlation data suggest that these events are confined to the extent of the mined out longwall and cluster close to the edges of the active longwall at the depth of active mining. These events occur in lockstep with the longwall advance and exhibit a high $b$ value of the Gutenberg-Richter relation (GR) of about 1.5 to 2.5 and consist of small magnitude events. Thus, these events represent the immediate energy release adjacent to the mined out area.

The second group consists of clusters located either slightly above or below the depth of active mining and occurring at the current position of the longwall face within the confines of the longwall. They consist of generally stronger events and do not follow GR. This activity might be linked to the failure of more competent layers above and below the mined out seam resulting in larger magnitude events.

Finally, one cluster represents seismic activity with a rather low $b$ value below 1 and events located partly towards the north of the longwall which are delayed with respect to the advance of the longwall face. These events are interpreted as brittle failure on pre-existing tectonic structures reactivated by the mining activity. 


\section{HAMNET - a temporary, local network for seismic monitoring of mining induced seismicity}

It is well known that mining activity has the potential to cause minor earthquakes up to local magnitudes of about $M_{\mathrm{L}}=3.0$ (see e.g. Gibowicz et al., 1990). Due to the shallow depth of this activity events with $M_{\mathrm{L}} \geq 1.2$ might be felt in the vicinity of a particular mine. Therefore, the monitoring of induced seismicity in the densely populated Ruhr region of Germany has a long tradition going back to the beginning of the last century when Mintrop installed the first seismological observatory in Bochum in 1908 (Mintrop, 1909a, b). Since the early eighties of the last century seismicity due to deep coal mining in the Ruhr area is routinely monitored by the seismological observatory at the Ruhr University Bochum. Routine analysis is published in annual reports and includes the determination of epicentre locations by a combination of array techniques and local stations in the mining areas as well as the local magnitude estimation (Fischer, 2013). Analysis of the frequency-magnitude distribution (FMD) of the entire data set revealed a $b$ value of around 2, which indicates a stress relaxation in the wake of the mining operation dominated by lower magnitude events (Bischoff et al., 2010a).

In order to study seismic activity induced by a single longwall operation in more detail a temporary seismic network was operated between June 2006 and July 2007 (Bischoff et al., 2010c). The monitored longwall panel S109 has a spatial extent of about $1000 \mathrm{~m} \times 300 \mathrm{~m}$ and is located at a depth of about $1100 \mathrm{~m}$. The exploitation of the coal seam was performed from August 2006 until April 2007. The seismic network called HAMNET consists of 15 three-component surface stations ( 6 broadband, 9 short period, see Fig. 1). Besides one station installed in the field, all stations were installed on the basements of private houses and public institutions which assured a good coupling to the subsurface. To improve the field installation this station was buried about one metre in the soil. In addition, the broadband stations were thermally isolated. Single event locations were determined by Bischoff et al. (2010c). Using $P$ onsets and a homogenous average velocity model they obtained high quality locations for 7337 events, which is equivalent to about 900 events per month. To determine the locations they used a homogenous velocity model which had been calibrated by a ground truth event with a known hypocentre, namely a rock burst event within the mine. The location accuracy was estimated by a grid search procedure for representative events and amounts to about $40 \mathrm{~m}$ in epicentre coordinates and $80 \mathrm{~m}$ in depth. Magnitudes range from $M_{\mathrm{L}}-1.7$ for very small events up to $M_{\mathrm{L}} 2.0$ for events that are considerably felt by the population. In their work magnitudes were calculated from the maximum amplitude of the displacement seismograms for each station, then averaged over the whole network and calibrated by comparison with the magnitudes of the regional network and catalogue. The resulting locations are shown together with the station network in Fig. 1

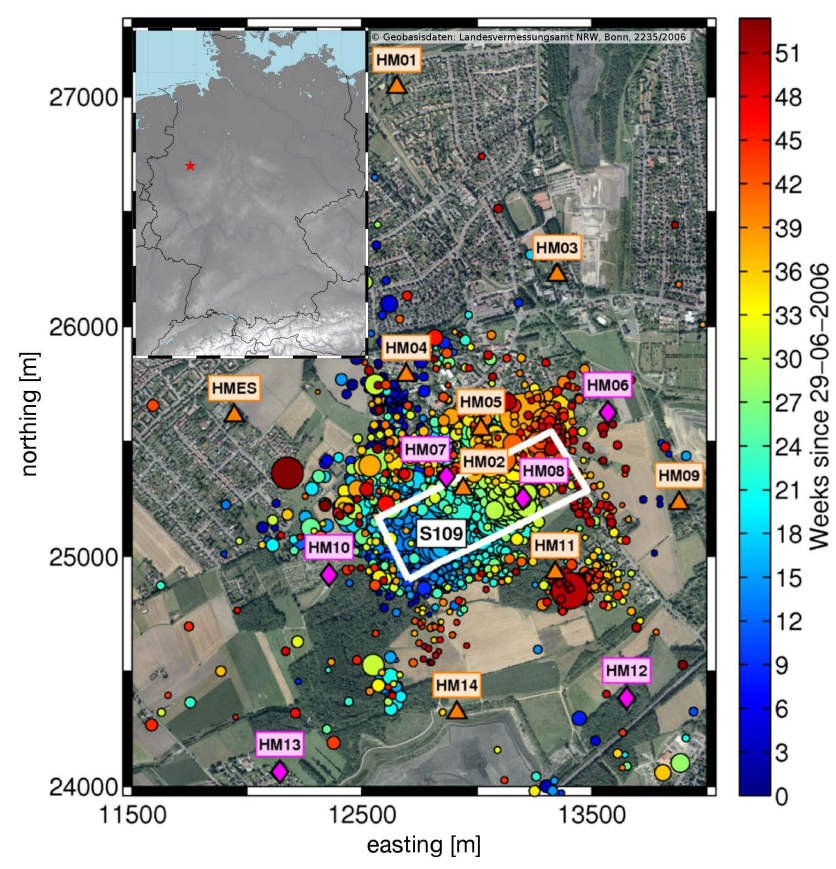

Fig. 1. Map showing the stations of the temporary seismic network HAMNET, the longwall S109 and the recorded seismic events. Diamonds denote broadband and triangles short period stations. Colours of the events depict the time of occurrence and the size of the circle scales with event magnitude.

and have been interpreted previously (Bischoff et al., 2010c) as follows. Seismicity mainly concentrates on the longwall panel S109 where mining operations are conducted and the adjacent broad area north of it, which is known as a tectonically prestressed region characterized by dipping of the coal seam and by tectonic faults. In addition, spatial clusters of induced events are active at some distance from the longwall panel, e.g. in the south near station HM14, southeast of the longwall near station HM11 and in the north around station HM04, where the blue colours indicate early seismicity that has been associated with the end of mining activity of the preceding longwall. Three factors controlling seismicity have been found: (1) longwall face seismicity (LFS) within the borders of the panel in close proximity to the ongoing excavation, (2) seismicity which spatially clusters at distances of up to $500 \mathrm{~m}$ from the longwall and (3) weak tectonic structures such as faults that lead to enhanced seismicity and preferred orientation of fault planes. Source mechanism studies of 105 larger events using $P$ wave polarities and $S$ polarization angles revealed two main types of rock failure (Fischer, 2010; Bischoff et al., 2010b). Fault plane solutions either showed steeply dipping normal faulting with strike parallel to the longwall face or vertical $P$ axes with variable strike. The first mechanism is associated with the failure of load-bearing sand-/siltstone layers which fail due to enhanced stresses during mining. The second source mechanism is interpreted as 
the failure of remnant pillar structures from previously mined out seams overlying the currently excavated seam or tectonically weak structures such as faults that are reactivated by the mining induced stresses. These findings are consistent with the proposed model of rock failure by Alber et al. (2009).

High inter-event waveform similarity has been observed for the HAMNET data set. A cluster analysis of these events based on their waveform similarity illuminates the internal structure of the event sequences which can be used to better characterize distinct processes in the seismogenic volume. Distinct clusters of events exhibiting similar waveforms might be caused e.g. by spatially distinct faults or different source mechanisms (e.g. Phillips, 2000; Maurer and Deichmann, 1995). To improve the understanding of the responsible mechanisms for brittle failure a correlation-based waveform-similarity analysis with subsequent cluster identification was performed. The correlation coefficient is sensitive to the event-receiver distance and possible disturbances of the waveforms due to local background noise. In order to suppress these effects a network correlation matrix was constructed. Furthermore, a new method to sort similarity matrices based on the similarity of adjacent rows of the respective matrix is introduced and applied to the HAMNET data. Such sorted similarity matrices have the advantage that they provide a quick visual overview as well as detailed information about the intrinsic properties of the data set. Such an analysis provides subsets of events having significantly different waveforms which could be used as master events for near real-time analysis of micro-seismicity in environments of induced seismic activity. Finally, distinct event clusters are extracted from the sorted similarity matrices by visual inspection. The obtained clusters are compared with the results of a standard single-linkage approach applied to the same original network similarity matrices. Additionally, relative relocations and frequency magnitude distributions for the different clusters are compared and reveal indications for different types of brittle rock failure for the induced seismic events.

\section{Network similarity matrices and matrix sorting}

\subsection{Establishing similarity matrices}

Clustering in general means accumulation of individual data to groups with members each sharing one or more properties with all the others. In seismology event clusters often indicate that individual earthquakes occurred at the same location or at the same time. Such sequences can be e.g. main-shock after-shock sequences or earthquake swarms (e.g. Hemmann et al., 2000; Becker et al., 2006). Clustering of mininginduced seismicity as discussed in this work provides information about the underlying processes leading to rock failure in the vicinity of a mine. The $\lambda / 4$-criterion implies that similar waveforms that have been recorded at one station originated within a distance of one fourth of the signal's dominant wavelength (Geller and Mueller, 1980). Additionally, waveform similarity also requires a similarity of the source mechanisms as proposed by Got and Fréchet (1993). Thus, in order to obtain clusters of seismic events with similar source mechanisms and close spatial proximity an algorithm quantifying waveform similarity is needed. The similarity of two time series $A_{a}$ and $A_{b}$ can be measured by the cross-correlation function $\mathcal{C}(\tau)_{a b}$, where $\tau$ is the time lag and indices $a$ and $b$ denote the two correlated time series.

$$
\mathcal{C}_{a b}(\tau)=\frac{\sum_{i=1}^{N} A_{a}\left(t_{i}\right) A_{b}\left(t_{i}+\tau\right)}{\sqrt{\sum_{i=1}^{N} A_{a}\left(t_{i}\right)^{2}} \cdot \sqrt{\sum_{i=1}^{N} A_{b}\left(t_{i}\right)^{2}}}
$$

As seismic recordings mostly consist of waveforms from three components, the discrete normalized cross-correlation $\mathcal{C}(\tau)_{a b}$ is modified to gain a measure of similarity incorporating the whole seismic information available. Thus, the formula used to establish the similarity matrices in this study is given by

$$
\mathcal{C}_{a b}(\tau)=\frac{\sum_{k=1}^{3} \varepsilon_{k}^{2} \sum_{i=1}^{N} A_{a k}\left(t_{i}\right) A_{b k}\left(t_{i}+\tau\right)}{\sqrt{\sum_{k=1}^{3} \varepsilon_{k}^{2} \sum_{i=1}^{N} A_{a k}\left(t_{i}\right)^{2}} \cdot \sqrt{\sum_{k=1}^{3} \varepsilon_{k}^{2} \sum_{i=1}^{N} A_{b k}\left(t_{i}\right)^{2}}},
$$

where $k$ denotes the recorded seismic component (in general either N-S, E-W or up-down) and $i$ each sample of the time series, $A$ means amplitude and $t$ time. The coefficient $\varepsilon$ allows weighting of each component separately. Adjustments of the factor can be necessary if the $H / V$-ratio at a single station is significantly different from others. Moreover, this factor can be utilized to increase the weight of single phases during analysis. As most of the S-wave energy is observed on the horizontal components, this phase could be highlighted by weighting the horizontal components. In the present work the weighting parameter $\varepsilon$ has been set to 1 for all components $k$, which implicitly means down-weighting the vertical component.

From the cross-correlation function the maximum coefficient and the corresponding time lag $\tau$ was retained for further analysis. The similarity matrix (SM) (e.g. Fig. 2, see also Eq. 2) and the lag matrix for a given event catalogue are symmetric and anti-symmetric matrices of dimension $L$, respectively. $L$ denotes the size of the catalogue. These contain the maximum cross-correlation coefficient of $\mathcal{C}_{a b}(\tau)$ and the corresponding time lags $\tau$ for each event pair, respectively.

$$
\mathbf{B}=\left(\begin{array}{cccc}
b_{1,1} & b_{1,2} & \cdots & b_{1, L} \\
b_{2,1} & b_{2,2} & \cdots & b_{2, L} \\
\vdots & \vdots & \ddots & \vdots \\
b_{L, 1} & b_{L, 2} & \cdots & b_{L, L}
\end{array}\right)
$$




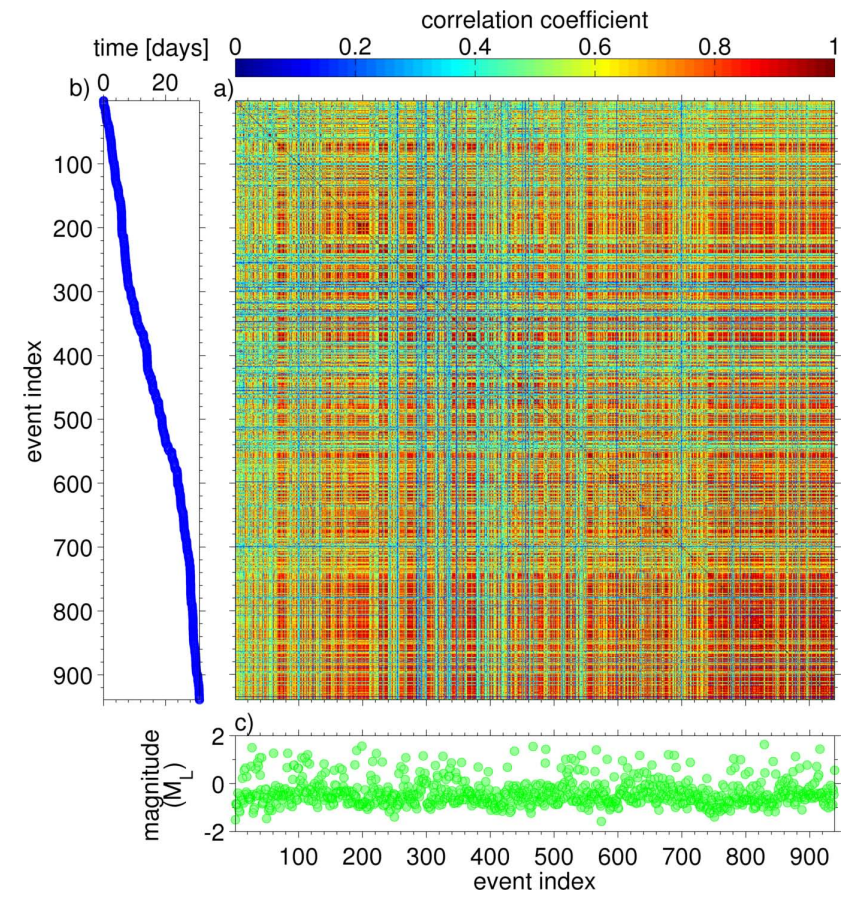

Fig. 2. Chronologically ordered similarity matrix for event data of October 2006 and station HM10 (a). (b) and (c) provide additional information on the time of occurrence and the magnitude distribution, respectively.

Diagonal elements of the matrix have values of one as they are the maximum coefficients of the auto-correlation function for all events with a corresponding time lag of zero.

Prior to cross correlation, data have been low-pass filtered with a corner frequency of $f_{u c}=20 \mathrm{~Hz}$. This was done because high-frequency content in the waveforms tends to artificially lower the cross-correlation coefficients due to scattering. This effect has been revealed by inspection of amplitude spectra of the records of two stations that showed increasing similarity with increasing event to station distance and vice versa (see Fig. 3). In addition to the low-pass filter, data of station HM13 have also been high-pass filtered with a corner frequency of $f_{l c}=1 \mathrm{~Hz}$ because data of this station show high amplitude long-period noise which eventually corrupts inter-event similarity at this site. In order to minimize the effect of temporary noise at a single station and to overcome the problem with the distance dependence of the cross-correlation coefficient a network matrix representing inter-event similarities over the total number of events in the data set has been calculated as the arithmetic mean of the single station SMs. The network matrix is initialized as an empty matrix of the dimension of the entire data set. Each row of the matrix belongs to a particular event. For each station SM the corresponding event row is searched and added to the network matrix. Additionally, a second temporary matrix counts the number of stations that contributed to each event pair in the network matrix. Finally, the network ma- trix is element-wise divided by the temporary count matrix. This approach is able to find similar events even in time intervals during which a single station might exhibit unusually bad noise conditions or might even be out of operation. Thus, a cluster analysis can be performed for the entire data set with one single matrix.

\subsection{Sorting of similarity matrices}

The idea of sorting the SMs in order to obtain clusters of similar events is that if the pattern of similarity of two events to all the others is similar, these two events have to be similar to each other as well. Thus, sorting the matrix by similarity of the rows gathers all events belonging to a cluster. Since the SMs are symmetric it is sufficient to sort for either rows or columns. In order to stabilize the sorting procedure the SM can be smoothed or sharpened before sorting. Therefore, the individual values of the SM are exponentiated by the smoothing parameter $\xi$. Values higher than one increase the contrast in the SM and thus result in more clusters with less members than without smoothing the SM. The opposite case holds for smoothing parameter values $0<\xi<1$.

The algorithm starts by finding the particular event which exhibits the highest similarity with respect to all the other events, i.e. the row of the SM having the largest cumulative sum. This can be written as

$\boldsymbol{i}_{\text {sort }, 1}=m_{0}$, where $\max _{m=m_{0}}\left(\sum_{n=1}^{L} b_{m, n}^{\xi}\right)$.

This events row index $m_{0}$ is the first entry of an index vector, the so-called sorting vector $\boldsymbol{i}_{\text {sort }}$, which is subsequently utilized to establish the sorted SM. $m$ and $n$ are the row and the column indices of $B$, respectively. Consecutively, the event exhibiting the highest similarity with respect to its sequence of cross-correlation coefficients (similarity pattern of the rows) to the previously found is searched for. Therefore, the similarity of two rows of the SM is calculated by cross correlating them without admitting any shift of the respective rows $m$. In case the cross-correlation coefficient is not normalized, this is mathematically identical to the scalar product of the row vectors (see also Maurer and Deichmann, 1995). All other entries of the sorting vector are iteratively found in this manner.

To prohibit fanning out of the afterwards sorted SMs during the sorting procedure and to make the process more stable, the $K$ most recently sorted event rows can be averaged prior to correlation. For increasing values of $K$, clusters tend to be larger and individual members exhibit smaller similarity values. This is comparable to the effect of applying lower threshold values of the cross-correlation coefficient for the single-linkage method (see below). For too high values of $K$, fanning out of clusters recommences (see Fig. 4). In case of the present data set a reasonable value for $K$ was found to be 2 by visual inspection of the sorted SMs after applying different $K$ values. In order to obtain a formulation which is 


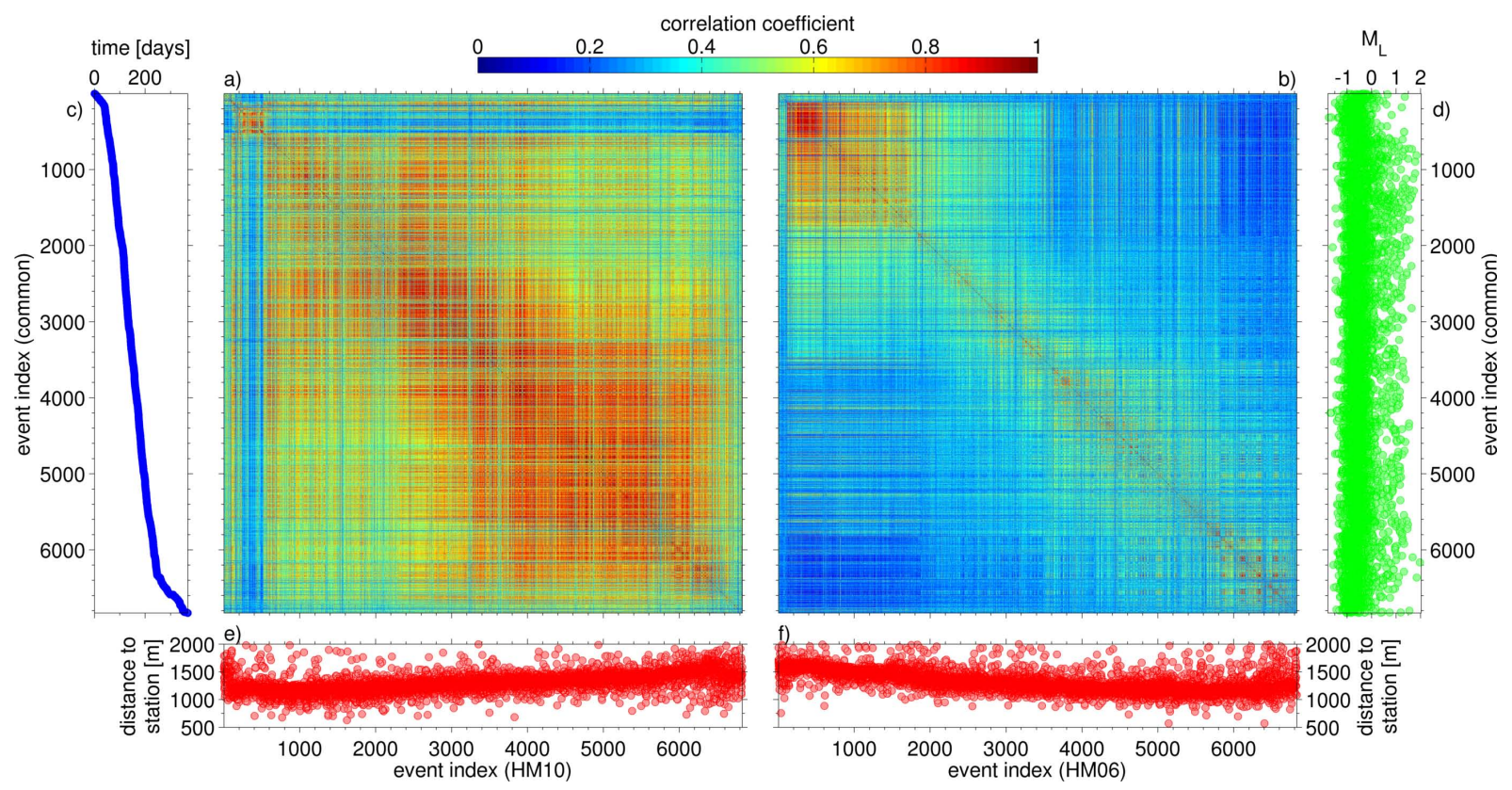

Fig. 3. Similarity matrices for two stations at opposing ends of the longwall. Event migration is away from HM10 (a) and towards HM06 (b) which were situated at the southwestern and the northeastern end of the longwall panel, respectively (see Fig. 1). (c) and (d) show the temporal and the magnitude distribution of the events. (e) and (f) depict event to station distance for the particular station-event combination, indicating a positive correlation between correlation coefficient and station-event distance.

also valid for the first few sorting steps where less than $K$ rows are already sorted, $K^{*}$ is introduced with

$K^{*}=\left\{\begin{array}{ll}K & \text { if } K \leq(L-\hat{m}) \\ (L-\hat{m}) & \text { if } K>(L-\hat{m})\end{array}\right.$.

$\hat{m}$ denotes the not yet sorted rows of the smoothed SM $\tilde{\mathbf{B}}=$ $b_{m, n}^{\xi}$ and $L$ the total number of rows in the matrix.

The correlation formula for all remaining events $\hat{m}$ reads then as follows:

$\boldsymbol{i}_{\text {sort, }(L-\hat{m})+1}=m_{0}$, where $\max _{\hat{m}=m_{0}}\left(\sum_{n=1}^{L} \sum_{k=1}^{K^{*}} \frac{b_{\hat{m}-k, n}^{\xi}}{K^{*}} \cdot b_{\hat{m}, n}^{\xi}\right)$.

This means looking for the row $m_{0}$ which maximizes the scalar product between itself and a sequence obtained by component-wise arithmetic averaging of the $K^{*}$ most recently sorted rows, i.e. the row exhibiting the pattern most similar to the average of the $K^{*}$ most recently sorted rows.

Sorted SMs are then given by

$\mathbf{B}_{\text {sort }}=b_{\left(i_{\text {sort }, m}\right),\left(i_{\text {sort }, n}\right)}$.

This sorting algorithm is independent of the database and thus is applicable either to station or network matrices. SMs sorted by this approach reveal clustering of events at first glance as can be seen in Fig. 5. To provide additional information on the waveform similarity itself, the waveforms are plotted in image representation next to the SM. Waveforms are sorted in the same way as the SM and time-shifted with respect to the source time of the first event of a particular group of events (not clusters). The time-shift is calculated as the cumulative sum of the secondary diagonal of the lag matrix. The source time is reset if either the time-shift exceeds a value of $2 \mathrm{~s}$ or the cross-correlation coefficient of the present to the previous event falls below a threshold of 0.85 . The image representation maintains recognition of sub-clustering and retains information on the source properties of clusters but is only available for station SMs.

\section{Identified event clusters}

\subsection{Visual cluster extraction from sorted network similarity matrices}

In order to find clusters, the sorted network SM as obtained by application of Eqs. (1), (3) and (4) to the HAMNET data set is plotted as an image. Distinct clusters are evident as patches of high inter-event similarity (see Fig. 6). Boundaries of the patches identifying highly similar events are picked and all events included within these boundaries are associated to a particular cluster. For this visual cluster identification from the sorted network matrix no threshold value for the correlation coefficient is used but the squareness of the patches. This means that events are associated to a cluster if this is depicted as a square of high inter-event similarity in contrast to the surrounding events in the sorted SM. 


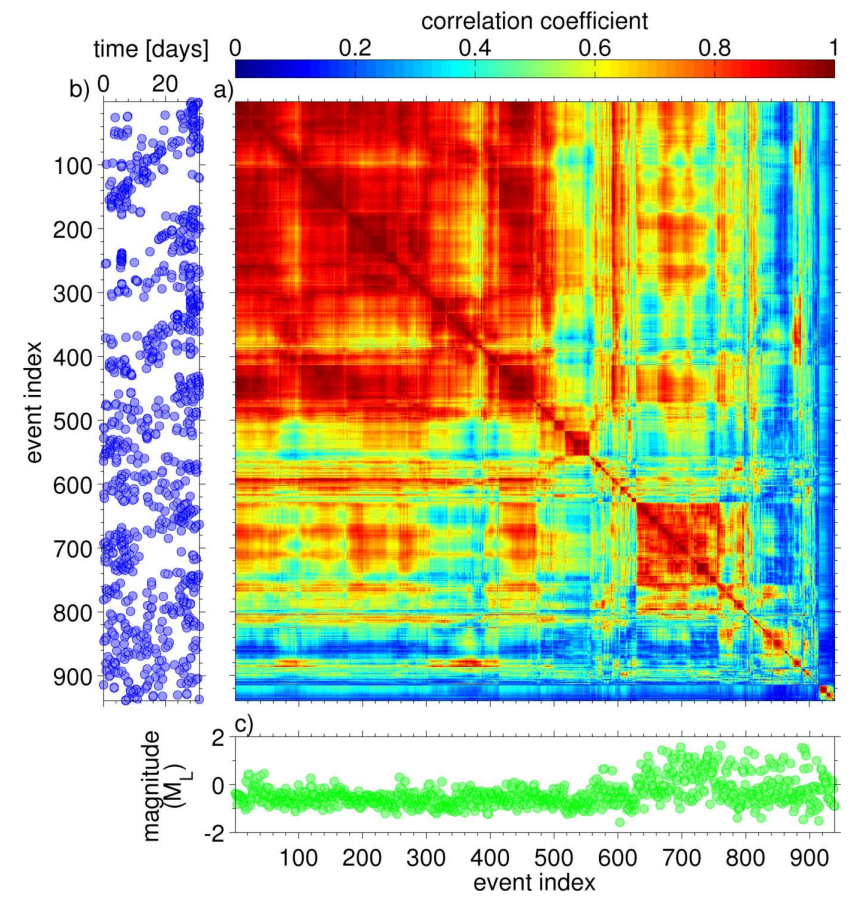

Fig. 4. Sorted similarity matrix (a) for station HM10 and October 2006. (b) and (c) provide additional information about time of occurrence and magnitude distribution respectively. Sorting of the matrix was performed with a value for $K$ of 5. In comparison to Fig. 5, obviously events are not consequently gathered. Instead high similarity patterns are fanned out, which is inconvenient for visual cluster extraction.

Figure 6 shows the sorted network SM for the whole HAMNET catalogue. Clusters are highlighted as patches of bright colours. Within the associated clusters there are obvious differences in inter-event similarity which are interpreted as sub-clustering. A total of 16 clusters which have more than 30 members are found, with nine of them having more than 70 members. The two largest clusters have 2545 and 1152 members, respectively, and thus make up more than half of the total data set. The eight largest clusters will be discussed in detail. Their locations and magnitude time distributions are shown in Fig. 7 (colour-coded) together with the events from very small clusters and unassociated events (grey).

From inspection of Fig. 7 it is apparent that the two largest clusters (black and blue) are clearly spatially distinct. Locations of the events forming the largest cluster (black) are limited to the northeastern part of the longwall panel while the events of the second largest cluster (blue) exhibit source locations in the southwestern part of the excavated area. On average the events of both clusters are at the mining level around $1100 \mathrm{~m}$. Furthermore, they consist mainly of small magnitude events not exceeding maximum values of $M_{\mathrm{L}}=1.2$ and $M_{\mathrm{L}}=0.5$ for the western and eastern cluster, respectively, and belong to the longwall face seismicity (LFS) described above.
In contrast, other large clusters (red, magenta and cyan in Fig. 7) located also within the geographic confines of longwall S109 exhibit strong events with maximum magnitudes up to $M_{\mathrm{L}}=1.8$. Their magnitude distributions show a much higher ratio of larger to smaller magnitudes. Although there is some overlap in their epicentral locations these clusters show a general tendency to occur in distinct regions. Depths of the respective events tend to be on average either a bit shallower (red, orange) or a bit deeper (cyan) than those of the largest clusters discussed previously. The red cluster nearly covers the same E-W extent as the largest cluster with the smaller magnitudes (black) but is limited to the northern part. The hypocentres are more shallow hinting at events that occur above the mining. The magenta cluster shows a comparable magnitude distribution including strong events. It is located at the same more shallow depth, but in the western part of the longwall panel. In contrast to the red cluster, it spans the entire $\mathrm{N}-\mathrm{S}$ dimension, similar to the epicentres of the events with smaller magnitudes at the mining level (blue cluster). The cyan cluster is concentrated at the southeastern border of the longwall panel and in slightly greater depths presumably below the active mining. Also these three clusters belong to the LFS.

One further cluster (orange) with higher magnitude events is concentrated at the northern border of the longwall panel. Whereas events of all previously discussed clusters are more or less confined by the outline of the panel the orange cluster includes epicentres clearly north of it. This observation suggests that events of this cluster do not belong to the LFS. Two additional clusters with small magnitude events are observed at the northeastern end of the longwall (yellow) and in the south (green) where the preparation of the following mining started towards the end of the observation period. The locations of the other identified clusters are not shown because they exhibit too few events to make reasonable statements with respect to their magnitude distribution or their spatial extent.

\subsection{Clusters found with the single-linkage method}

In order to compare the clusters found with the new sorting algorithm and the visual inspection the results are compared to those obtained by the well known single-linkage (SL) clustering or equivalence class algorithm. This is a simple, straightforward scheme to organize seismic events based on their waveform similarity into clusters (e.g. Aster and Scott, 1993; Everitt, 1993). In this approach each event starts out as its own single-member cluster and two distinct clusters are merged into one larger cluster containing all events of the previously distinct clusters whenever any two events from the different clusters possess a similarity value above a defined threshold. As a consequence, the newly formed cluster may exhibit similarity values well below the required threshold value for event pairs. For a gradual change of the similarity values (i.e. the waveforms) in an earthquake data set this may 


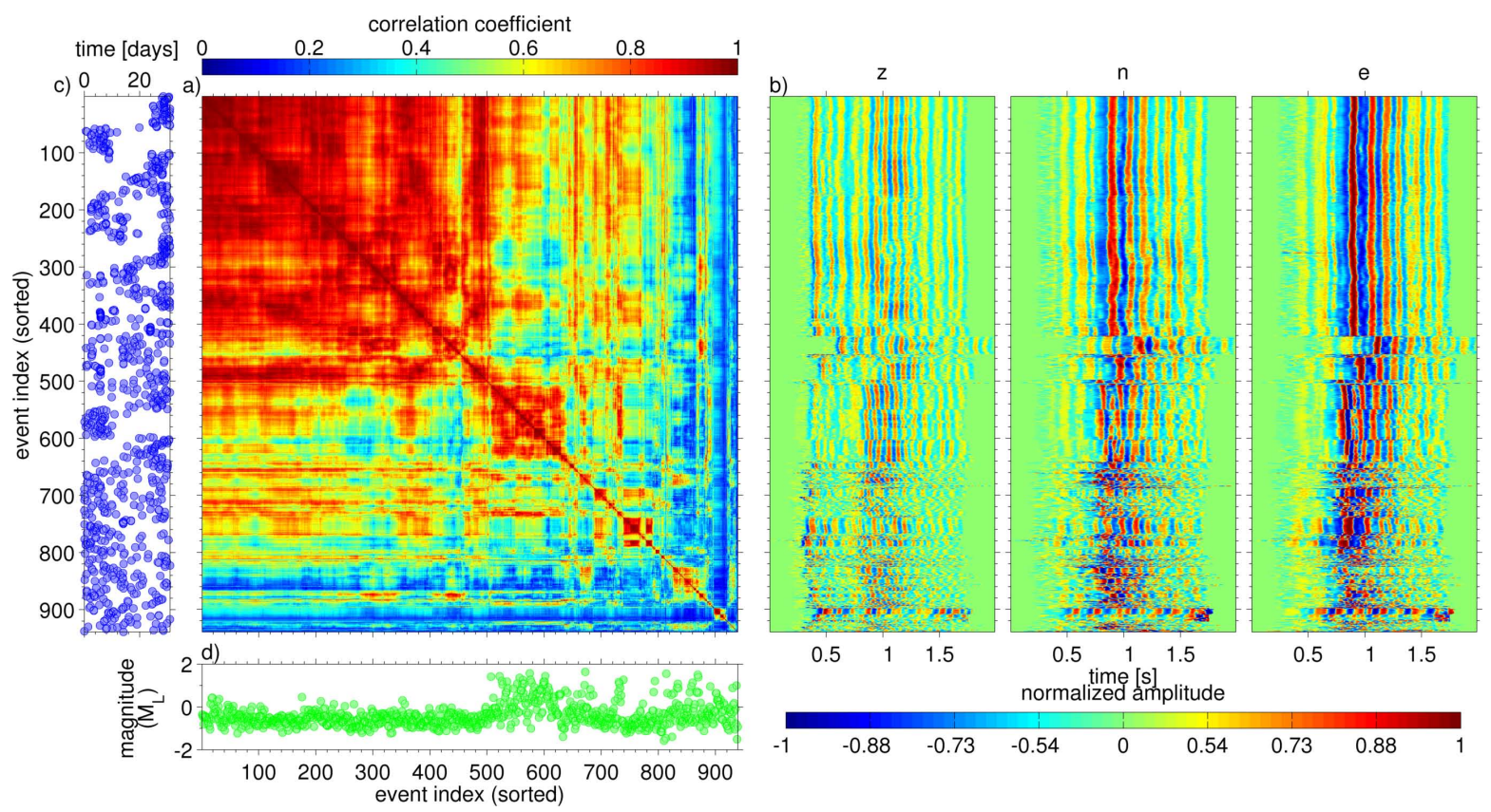

Fig. 5. Sorted similarity matrix (a) for station HM10 and October 2006 with the corresponding waveforms (components $Z, N, E$ ) which have been correlated (b). (c) and (d) provide additional information about time of occurrence and magnitude distribution respectively.

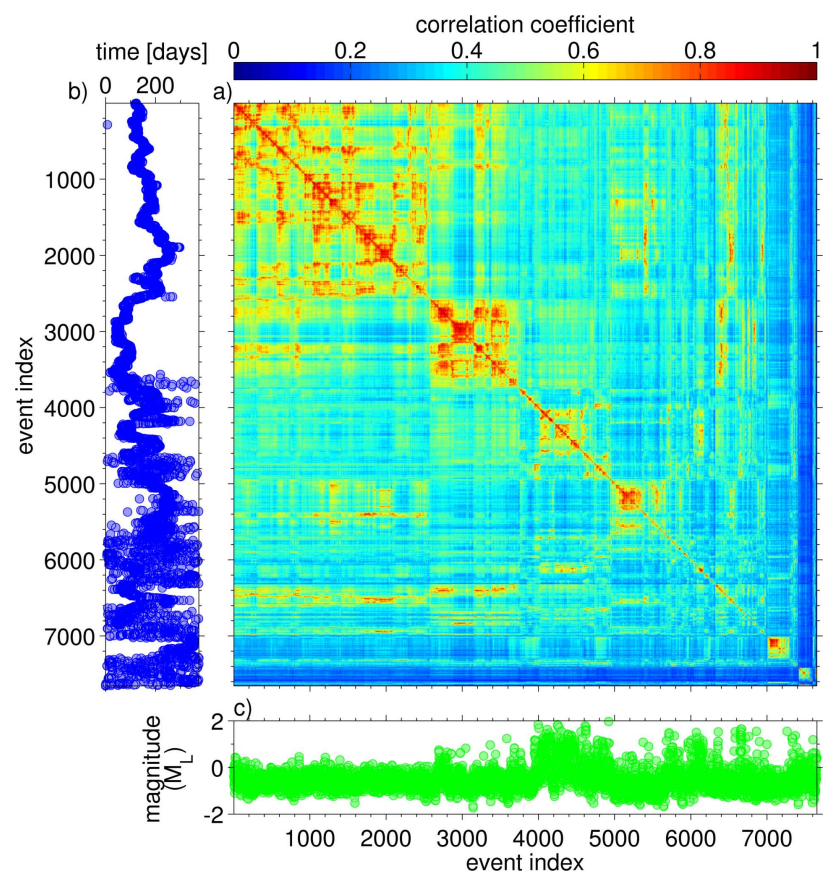

Fig. 6. Sorted network similarity matrix with associated event clusters found by visual inspection. Clusters finally identified are indicated by the bright colours close to the diagonal of the SM. The largest clusters correspond to those shown in Fig. 7.

result in spatially extended clusters where two events from opposing ends of the cluster exhibit considerable differences in their waveforms.
For the investigated HAMNET data set the similarity value used for an event pair is the weighted cross-correlation coefficient described in the previous section, which was also used for the SM sorting algorithm and visual cluster extraction. The chosen threshold value for the single linkage cluster association was 0.9 based on the trade-off between the creation of new clusters and the coalescence of existing clusters when gradually decreasing the cross-correlation value. This threshold value results in the identification of 188 distinct clusters with a maximum of 1696 cluster members in one case and with eight clusters having more than 70 members. However, most of the clusters are small with only a few members and 160 of the clusters have 10 or less members. Most of the largest clusters found with the SL approach can be associated to the largest clusters obtained by the matrix sorting algorithm. Especially, the two largest clusters of the SL approach correspond to the two largest clusters of the matrix sorting approach. Furthermore, the large clusters with higher magnitudes identified by the new approach (red, magenta, cyan and orange in Fig. 7) can be found also in the SL approach (same colours in Fig. 8). The only difference among the eight largest clusters of both approaches are the two clusters with small magnitude events (yellow, green) found by the sorting algorithm. While the green cluster was also found in the SL approach, albeit with too few members to belong to the eight largest clusters, the yellow cluster was partly incorporated in the largest cluster (black) found by the SL approach.

In general, the chosen threshold value of 0.9 for the SL approach tends to create clusters comparable to those of the sorting algorithm. However, the corresponding clusters in the 

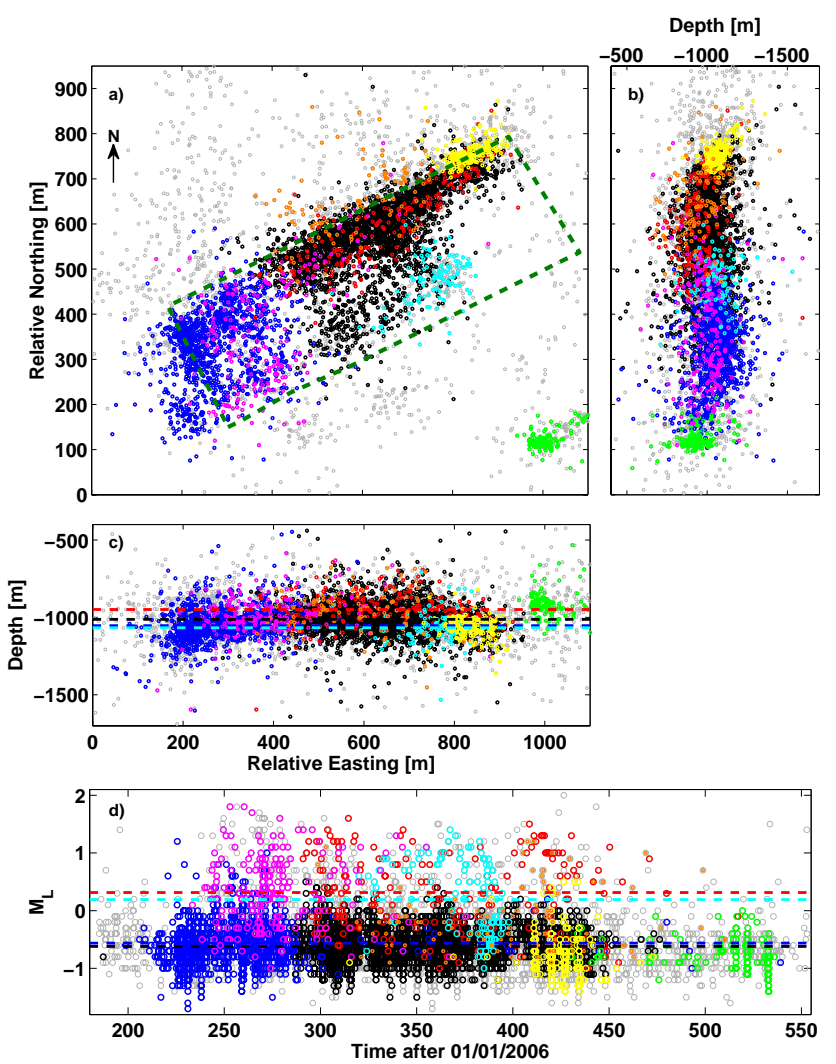

Fig. 7. Result of the visual cluster extraction (absolute event locations). Colour coded are the eight largest clusters found by visual inspection of the sorted network SM in (a) map view, (b) $\mathrm{N}-\mathrm{S}$ depth section and (c) E-W depth section. (d) Magnitude over time plot of the events. In all figures the grey circles indicate the events in the catalogue not belonging to the eight largest clusters. Broken line in (c) indicates the mean depth of the respective cluster. Broken line in (d) indicates the mean magnitude of the respective cluster. For clarity only the mean values of the four largest clusters are depicted in (c) and (d). Note the exaggeration of the N-S and E-W axes in (b) and (c), respectively, with respect to the depth axis.

SL approach have fewer events. This discrepancy cannot be remedied by simply lowering the threshold value because this leads to a coalescence of distinct clusters before they reach the event numbers observed in the sorting algorithm. Thus, no SL threshold could be found to associate the same events to the clusters.

\section{Event relocation and frequency magnitude distributions}

\subsection{Relative event relocation}

In order to improve the relative locations between events, relocation procedures like the master event technique (e.g. Kraft et al., 2006; Deichmann and Giardini, 2009), the joint hypocentral determination (e.g. Douglas, 1967; Pujol, 1988)
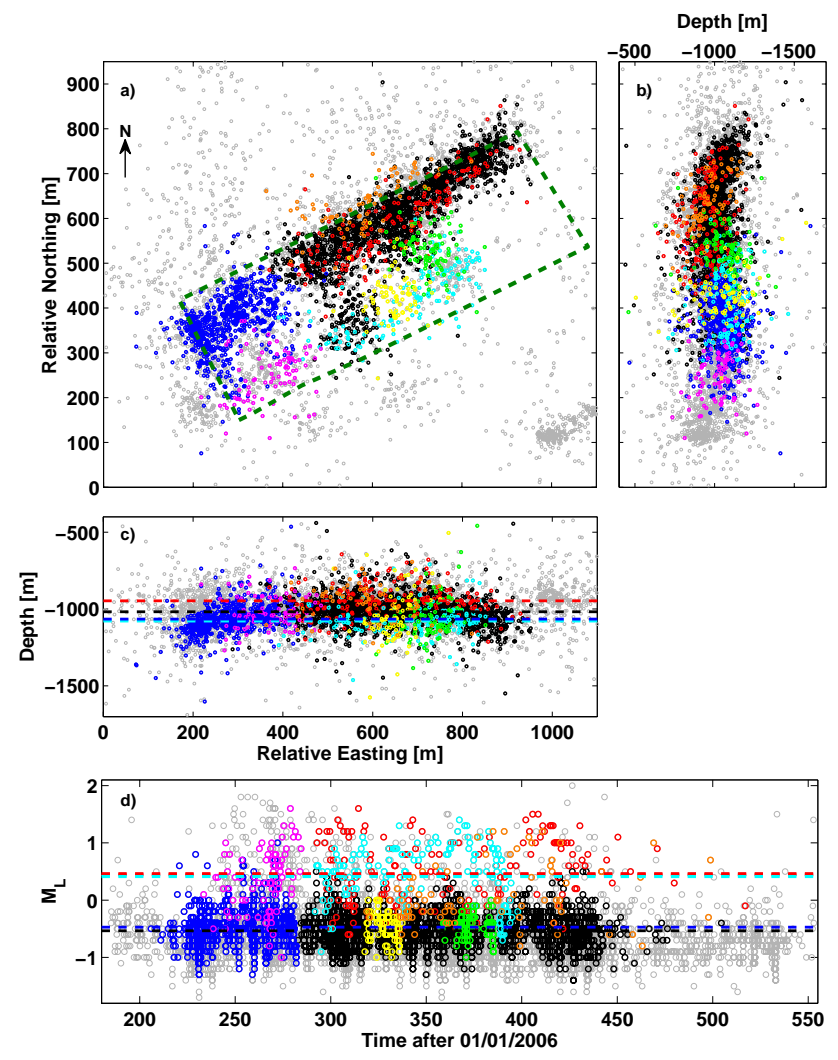

Fig. 8. Result of the single linkage cluster analysis (absolute event locations). Colour coded are the eight largest clusters found with the single linkage approach in (a) map view, (b) N-S depth section and (c) E-W depth section. (d) Magnitude over time plot of the events. In all figures the grey circles indicate the events in the catalogue not belonging to the eight largest clusters. Broken line in (c) indicates the mean depth of the respective cluster. Broken line in (d) indicates the mean magnitude of the respective cluster. For clarity only the mean values of the four largest clusters are depicted in (c) and (d). Note the exaggeration of the N-S and E-W axes in (b) and (c), respectively, with respect to the depth axis.

or the double difference method (Waldhauser and Ellsworth, 2000) can be applied using either travel times from catalogue data, waveform cross correlation or a combination of both. By applying these techniques, effects of the wave path outside the restricted source region of the events which are relocated with respect to each other are eliminated and all observed travel-time differences between the events are effectively translated into hypocentral distances between these events assuming a homogeneous velocity model in the source region (e.g. Wolfe, 2002). In this study the hypoDD double difference relocation code of Waldhauser and Ellsworth (2000) is used. Residuals between observed and theoretical travel-time differences, i.e. double differences, are iteratively minimized with a least-squares solution in this approach. This is done for pairs of earthquakes at each station while linking together all available event-station pairs 
(Waldhauser and Ellsworth, 2000). In consequence, each single earthquake contained within a data set is then relocated with respect to all other events of this data set with which it shares either catalogue or cross-correlation travel-time information.

For the relocation procedure only $P$ phases were used in analogy to the single event location scheme because picking the $S$ phase is difficult and often speculative due to the complex character of the waveforms. However, restricting the analysis to $P$ phases leads generally to satisfactory results when high precision travel-time differences for a sufficient number of stations can be obtained by means of cross correlation (e.g. Waldhauser and Ellsworth, 2000). Due to the fact that the previous cluster analysis identified a large number of events with highly similar waveforms a relative relocation of these events seems promising.

While relative positions between the different clusters as well as positions of isolated events and events belonging to smaller clusters are controlled by catalogue travel-time data, cross-correlation travel-time information is used for events belonging to the eight largest clusters found with the SM approach. This allows a closer inspection of the relative spatial distribution of events belonging to the same cluster. Thus for each event belonging to one of the eight largest clusters cross-correlation travel-time differences were calculated with respect to the 30 nearest neighbours belonging to the same cluster. Relative $P$ phase travel-time differences for an event pair at the same station are calculated in the time domain using time windows of $0.4 \mathrm{~s}$ length centered on the manually determined $P$ onset. For cross correlation the data is filtered with a 3rd order zero-phase Butterworth filter between 2 and $50 \mathrm{~Hz}$ after being resampled to $2000 \mathrm{~Hz}$ in order to improve the temporal resolution of the cross correlation. Due to the fact that the event-station distance is except for very shallow events at least $1 \mathrm{~km}$ the data window only contains the $P$ and not the $S$ onset. Only event pairs with a normalized cross-correlation coefficient of at least 0.8 were kept and used for the relocation procedure and the weighting coefficients of the differential travel times were chosen to be the squared values of the cross-correlation coefficient. In total 1325602 cross-correlation $P$ travel times and 1526325 catalogue differential travel times were used to relocate 6867 events remaining from the original 7337 events after matching the differential travel times.

For the hypoDD relocation a minimum of eight catalogue differential time observations per event pair was demanded in the relocation procedure and hypoDD was run with a five step weighting scheme with increasing emphasis on the crosscorrelation data and tighter spatial control on the travel-time information used, starting from the network hypocentres. The same homogeneous half-space velocity model as used for the single event location $\left(v_{p}=3.88 \mathrm{~km} \mathrm{~s}^{-1}\right)$ was applied. In the final step a maximum event separation of $100 \mathrm{~m}$ for cross-correlation data and $150 \mathrm{~m}$ for catalogue data with a five times larger weighting of the cross-correlation information was chosen.

The locations of the 6783 events retained after relocation are depicted in Fig. 9. Nearly 100 events lost linkage during the iterative reweighting process due to distance cutoff in the case of large event separation or to outlier removal due to high data residuals and were subsequently deleted. When compared to the original locations (Fig. 7) a much tighter clustering of the events can be observed. This is especially true for the events belonging to the largest clusters because for their internal event relocation high resolution crosscorrelation travel times were used. Events belonging to the largest clusters associated with longwall S109 are now much more tightly confined to the outline of the longwall. The only exception is the orange cluster in Fig. 9 whose events tend to locate towards the north of the longwall. Furthermore, the events linked to the longwall S109 exhibit a clear tendency to cluster at the northern border of the longwall and to a lesser degree at the southern border after relocation when compared to the single event locations (Fig. 7). The E-W extent of the relocated seismicity of longwall S109 is more or less the same as that of the single event locations and no significant further spatial concentration of events belonging to the largest clusters is observed in that direction. Although some increase in the spatial clustering is observed for the events not belonging to the largest clusters, this is less obvious. However, this is expected because only catalogue information was used to constrain the locations of these events. The epicentral clustering (Fig. 9a) is much more pronounced than the depth clustering (Fig. 9b, c), indicating a better control on the horizontal coordinates than on the vertical one. However, the observation that the large magnitude clusters located on average at depths above or below the level of the small magnitude clusters is supported by the results of the relocation.

\subsection{Frequency magnitude distributions}

The $b$ value of the frequency magnitude distribution (FMD) describing the ratio of small and large events in an earthquake population is now known to show considerable spatial and temporal variation (e.g. Wiemer and Schorlemmer, 2007; Wiemer and Wyss, 2002). This global-scale as well as localscale variability seems to depend on the acting stress regime (e.g. Schorlemmer and Wiemer, 2005; Becker et al., 2010), the magnitude of the applied shear stress (e.g. Amitrano, 2003) and possibly also the material involved in the failure process (e.g. Köhler et al., 2009). Furthermore, it is well known that FMDs in the mining environment often exhibit a bimodal character (e.g. Bischoff et al., 2010a; Richardson and Jordan, 2002; Gibowicz and Kijko, 1994) with small magnitude events believed to be concentrated around the active mining, indicating the almost instantaneous stress release in the immediate vicinity of the mining level while larger magnitude events are linked to pre-existing geological 


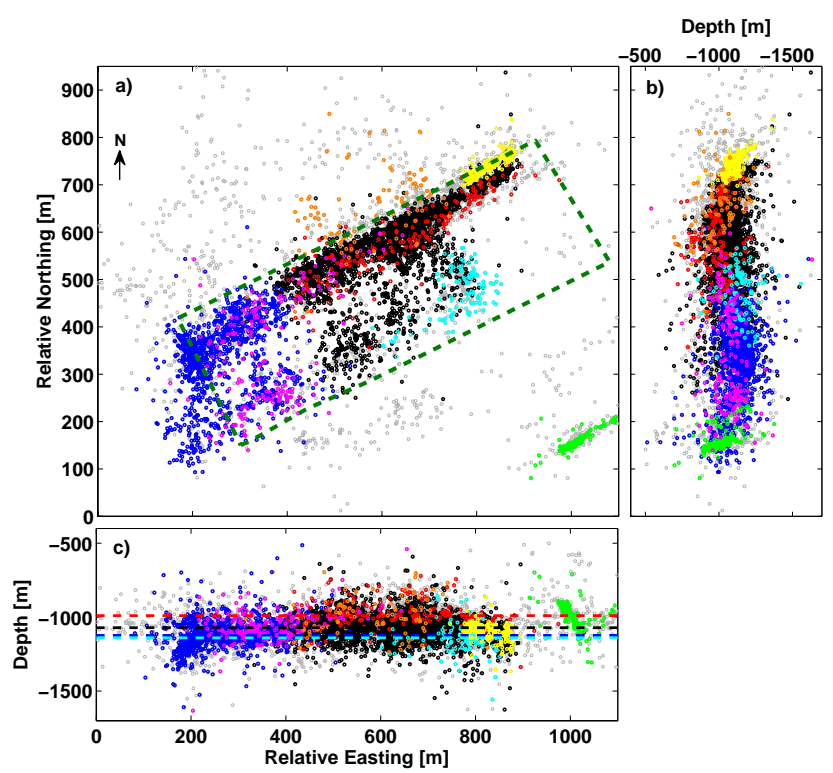

Fig. 9. Result of the double difference relocation of the events depicted in Fig. 7 in (a) map view, (b) N-S depth section and (c) $\mathrm{E}-\mathrm{W}$ depth section. The largest clusters discussed in the text are plotted in colour according to the previous figures. The remaining events of the catalogue are depicted in grey.

features like faults or competent layers (e.g. Richardson and Jordan, 2002; Gibowicz and Kijko, 1994). Finnie (1999) showed that a careful data analysis can spatially separate these different event types.

In this study $b$ values are calculated using the maximum likelihood approach of Aki (1965) incorporating a correction for the binning width of the catalogue (Bender, 1983), which is 0.1 magnitude units in our case. Magnitudes of completeness are determined using the goodness-of-fit test (GFT) (Wiemer and Wyss, 2000) demanding an $R$ value at least below 10 but preferentially below 5 . The $R$ value describes the absolute difference in the number of events in each magnitude bin between the observed magnitude distribution and a synthetic power law distribution with parameters from the maximum likelihood estimation (see Wiemer and Wyss, 2000, for details). An $R$ value of 0 indicates a perfect fit to the assumed GR power law. FMDs with an $R$ value above 10 for all possible completeness magnitudes are regarded as non-GR distributions and accordingly no $b$ value is calculated for them. Presented $b$ values (Fig. 10) are mean $b$ values of 1000 bootstrap runs of the respective cluster and error bounds indicate the standard deviation of these bootstrap results.

The FMD for the complete HAMNET catalogue displays the typical bimodal behaviour of mining induced seismicity (Fig. 10). A clear bump in the distribution between about $M_{\mathrm{L}}=0.2$ and $M_{\mathrm{L}}=1.2$ indicates that the distribution does not follow a GR law (Gutenberg and Richter, 1944) and that subsequently no $b$ value estimation for the entire catalogue
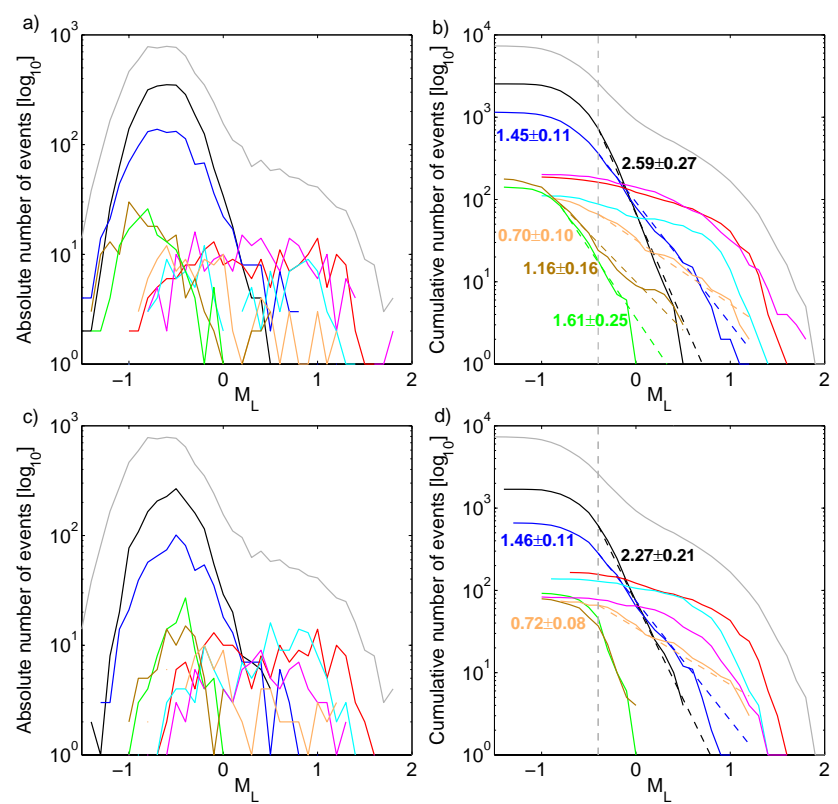

Fig. 10. (a) Absolute and (b) cumulative frequency magnitude distributions of the eight largest clusters found by visual extraction from the sorted network matrices. Colour coding is the same as in Fig. 7 except for the yellow cluster which is depicted in brown here for better visibility. (c) Absolute and (d) cumulative frequency magnitude distribution of the eight largest clusters found with the SL algorithm. Vertical broken grey line indicates the approximate completeness magnitude of the entire catalogue. Also indicated in (b) and (d) are the $b$ values for those frequency magnitude distributions exhibiting a GFT residual of less than $10 \%$ and having a completeness magnitude comparable or better than the entire catalogue.

is reasonable. However, it should be noted that by applying a goodness-of-fit criterion of $90 \%$ to the data, i.e. the residual between the observed and the theoretical distribution is less than $10 \%$ (see e.g. Wiemer and Wyss, 2000), a $b$ value of 0.88 and a corresponding completeness magnitude of $M_{\mathrm{L}}=-1$ is obtained. This apparent fit to the data is due to the large number of events and presumably the result of the superposition of two event types with very different FMDs, and stresses once again the point that even $b$ values which meet certain quality criteria should still be critically assessed.

The FMDs of the two largest clusters found with the different clustering approaches exhibit a clearly linear part above the magnitude of completeness with rather steep slopes and corresponding $b$ values of about 2.4 and 1.5 , respectively. Thus, there is also a large difference between their $b$ values although the events making up the two clusters appear at comparable depths and one might be tempted to assume the same mechanism for these events. The fact that the cluster occurring at the beginning of the mining activity in the western part of the longwall (blue) has the lower $b$ value corresponds to the observation that it exhibits higher magnitudes 
of up to $M_{\mathrm{L}}=1.2$ compared to the later cluster in the eastern part (black) with a maximum magnitude of $M_{\mathrm{L}}=0.5$. In contrast, the clusters with larger magnitude events of up to $M_{\mathrm{L}}=1.8$ (red, magenta, cyan in Fig. 10) which tend to be located slightly above or below the two largest clusters exhibit a broader peak in the FMD and an almost constant number of events in the magnitude range between the completeness magnitude of the catalogue and a magnitude of about $M_{\mathrm{L}}=1.2$. Thus, no $b$ value can be determined for these clusters because they do not follow a GR relation. Interestingly, the cluster of seismic events found towards the north of the active longwall (orange) exhibits a FMD following the GR law with a linear part above the magnitude of completeness and a rather low $b$ value of about 0.7 . Thus, this cluster exhibits a FMD characteristic clearly distinct from the two largest clusters with their high $b$ values and the other clusters with large magnitude events which show no GR behaviour.

\section{Discussion}

\subsection{Cluster association}

Maurer and Deichmann (1995) proposed a method for automatic cluster association. They used waveforms of $P$ and $S$ phases for cross correlation separately and afterwards combined the results. As the source-receiver distances in the research area of this work are very small, the SMs have been established from three-component seismograms containing both $P$ and $S$ wave energy and therefore also account for deviations in $(S-P)$ travel times. Subsequently, a change in $S-P$ time due to a different location will result in a lower crosscorrelation coefficient. The suggested algorithm to calculate the cross-correlation coefficients for a single station by the weighted sum of the three components of the recorded wavefield allows for user defined adjustments. Thus one might e.g. give the $P$ wave a stronger weight by giving the vertical component a higher weight than the horizontal ones in case the $P$ wave is the most prominent arrival on the vertical component.

Because it is often observed that different stations of a seismic network produce rather different patterns in their similarity matrices (Fig. 3) it has been decided to use a network SM for cluster identification. The construction of a network matrix is able to at least partly account for such problems as the distance dependence of the cross-correlation coefficient or spurious noise at single stations. The often observed distance dependence of the cross-correlation coefficient is attributed to the fact that signal components with high frequencies are strongly attenuated with increasing distance. Thus at larger distances from the seismic source only the low frequency components of the signal remain. Because the high frequency components of the signal transmit information about small differences in the seismic source processes, even slightly different sources will appear more similar when recorded at larger distances where the lower frequency signals dominate the wavefield. Constructing a network SM by averaging over all available single station similarity matrices in a network reduces this effect of distance dependence. Furthermore, the influence of temporary noise at single stations is reduced by using a network matrix. While a short noise burst at one station might result in a very low crosscorrelation coefficient for this event for this particular station the information from other stations exhibiting high similarity values will be incorporated in the network matrix and ultimately lead to the identification of this event as a cluster member. There are of course also other possible mechanisms to account for these problems. One might introduce e.g. a threshold value for the cross-correlation coefficient and only count the number of stations exhibiting a cross-correlation coefficient above this value. This would be one way to remove noisy station data from the analysis. To account for the distance dependence of the SM one might also use the data from a station exhibiting a high signal to noise ratio and somehow weight the cross-correlation coefficient with respect to the source-receiver distance. However, the approach to use an averaged network matrix incorporating all available information has the advantage that no parameters must be chosen and that the whole waveform information is included in the analysis.

Event clusters in the data set were identified visually from the network similarity matrices. In order to make this identification possible the network matrix is sorted automatically. In contrast to other sorting algorithms like SL or complete linkage which actually only consider the similarities between events belonging to the same cluster, the presented sorting algorithm incorporates information about all available events. This is achieved by calculating cross-correlation coefficients between the rows of the SM. The SM is then rearranged in such a way that rows being very similar to each other are located next to each other. In consequence this leads to a SM with visually apparent event clusters. Moreover, subclustering is evident at first glance whereas such an identification for the SL method requires a rerun with a different threshold value. However, it must be stressed that manual cluster determination from sorted SMs very much depends on the sorting parameters $K$ and $\xi$.

The averaging parameter $K$ has been introduced because the sorted SMs showed clusters but unfortunately these were not ultimately connected, which made it difficult to determine clusters of events manually. This phenomenon is called fanning out of the sorted SM in this work. Averaging the last two sorted rows prior to zero-offset correlation with the not yet sorted rows decreases this effect significantly and the visibility of complete clusters improves a lot. Smoothing the SM by exponentiation with $\xi$ prior to the sorting procedure influences the overall contrast of the SM and helps suppressing the influence of small variations in cross-correlation coefficients which cause the sorting algorithm to produce artifacts in the sorted SM. Here, it turned out that a smoothing 
parameter of $\xi=1.5$ gives the best results for all stations. It would also be possible to determine the best smoothing parameter for each station separately; however, for convenience this was not done in this work. Additionally, the final extracted clusters are somehow subjective because the analyst has to decide where exactly to draw the border between two different clusters.

The cluster association with an algorithm like the SL approach also depends on the somewhat subjective choice of the threshold value. Although some attempts were made to find an optimal value by considering the trade-off between the creation of new clusters and the coalescence of existing clusters (e.g. Aster and Scott, 1993) one might still find reasons to choose a higher or smaller threshold value in order to obtain smaller clusters with more similar events or larger clusters with events exhibiting a larger waveform variability, respectively. Due to the fact that the migrating mining activity produces a lot of events with gradually changing waveforms, a rather high threshold value of 0.94 results in the maximum number of equivalence classes which is sometimes regarded as the criterium for determining the optimum threshold value (e.g. Aster and Scott, 1993). At this value only about $23 \%$ of all events are associated with a cluster and only two clusters with more than 80 events exist. This makes the statistical comparison of single clusters, e.g. with respect to their FMDs or their inter-event distances difficult. Thus a lower threshold value of 0.9 with $51 \%$ of all events now associated with a cluster was chosen. The problem with the proper choice of the threshold value in the present case stresses the advantage of the cluster extraction from the sorted network matrix where the visual control also allows an assessment of the sub-clustering of the events. Especially, the separation of the two largest clusters found with both approaches is more stable in the sorting algorithm which keeps these clusters distinct; while the SL method merges these clusters when reducing the threshold value before they obtain as many members, as in the case of the visual extraction from the sorted SMs. That these two clusters might in fact be distinct is supported e.g. by their different FMDs (Fig. 10). However, the final cluster distribution obtained with the SL approach reasonably resembles the distribution found with the sorted SMs, although still less events were associated with a cluster than in the matrix sorting scheme.

\subsection{Relative event relocation}

Different filter as well as weighting parameters were applied to test the sensitivity of the relocation results to the chosen parameter values. Choosing a lower upper border for the frequency band used for filtering as well as a lower threshold for the cross-correlation coefficient results in the incorporation of travel-time information stemming from event pairs that exhibit considerable waveform variability. This did not lead to a significant improvement in locating events. Thus for the small spatial distances that the relocation wants to resolve also the high frequency content of the signal must be conserved.

The distance of the 30 nearest neighbours varies from 19 to $1160 \mathrm{~m}$ for the single event hypocentre locations but is for $95 \%$ of the events less than $200 \mathrm{~m}$ and thus much smaller than the distance from the events to the stations, which underscores the tight spatial clustering of the events. For the eight largest clusters discussed in detail the event separation is even smaller on average and the only exceptions are a few events showing large depth uncertainties (Fig. 9b, c). Due to the nature of the recorded seismicity which is distributed over a larger volume controlled by the progression of the mining activity it is not expected that all events tightly collapse on any single distinct linear or planar structure as often observed with tectonic events caused by sharply limited rupture surfaces (e.g. Waldhauser and Ellsworth, 2000). The general appearance of the relocated seismicity proved rather robust to moderate changes in the weighting parameters as well as the damping of the relocation procedure. Also varying the assumed velocity by $\pm 10 \%$ or using a layered velocity model did not significantly change the relocation results. The relocated events exhibited the largest sensitivity with respect to the bandpass filter used for calculating the cross-correlation travel times. However, several features of the relocated event distribution are rather stable. Events are mainly clustered at the northern edge of the longwall and to a lesser degree at the southern edge as well. In between these two lines of increased seismic activity exists a central zone with reduced event numbers. This observation might be explained by stress concentrations at the edges of the longwall as obtained by synthetic stress calculations for other coal mines (e.g. Al Heib, 2012). In this case the elevated activity at the edges of the longwall are a direct consequence of the current mining activity. The event distribution might also be influenced by the geometry of pre-existing longwalls in the vicinity of the current mining operation and thus be caused by stress changes due to a combination of current stress transients and already existing stress concentrations.

The depth resolution of the different clusters is not as much improved as the epicentral resolution by the relative relocation and thus it remains difficult to make definite statements regarding the relative depth location of the different clusters. However, the general trend that the clusters with larger magnitudes are located either slightly above or below the largest clusters is confirmed. This observation becomes even more obvious when only the best constrained events of the different clusters are compared (Fig. 12). If the velocities of the subsurface are varying on a small scale, the applied velocity model may in some cases not sufficiently represent the true subsurface. Then differences in the locations may be artifacts of the location procedure. Reasons for small-scale variations are, for example, local heterogeneities of the rock material or - which plays an important role in mines - local stress changes which are induced by the mining and may 
result in huge deviations from the assumed velocity. However, for the following reasons the influence of stress changes on our velocity model and locations are disregarded, respectively. Firstly, stations directly above the hypocentre control the depth determination. Thus, in this case, the different depths are supported by the network geometry and the high data quality. At least seven $P$ onsets for single event locations - more than 10 for the majority of the events - are available. The dense network including seven stations directly above the mining or at less than $300 \mathrm{~m}$ distance from the longwall panel ensures that onset times of stations above the hypocentre are used for the vast majority of the events. Secondly, one would expect to observe effects of local changes of the velocity model for clusters in different regions, i.e. with differing epicentres. In this case the clusters with only small magnitude events and the clusters including stronger events cover the same region. Therefore, the ray paths to the stations are nearly vertical and thus only the average velocity is relevant here and is identical for the more shallow and the deeper events. In summary, it is concluded that the centroids of the clusters are clearly separated by depths, although - as discussed above - the epicentres and thus the ray paths are similar. Thus, the observed separation at different depth levels at the mining level and above or below, respectively, is most likely a true characteristic of the observed seismicity.

While most of the identified clusters are well constrained by the outline of longwall S109 there exists one cluster at its northern border which shows also significant activity towards the north of the longwall (orange cluster in Figs. 7, 8, and 9). This cluster is found by both clustering methods, although with slightly different members, and it seems unlikely that this northward offset of the events is only an artifact of the relocation procedure. This behaviour is already visible in the single event locations indicating seismic activity not exclusively confined to the outline of the longwall. From inspection of Fig. 9 it is obvious that the large cluster active at the beginning of longwall mining (blue) also exhibits events towards the west of longwall S109. These events are most likely linked to working activity in the passageways and corridors leading to the mined longwall. Their occurrence outside the confines of longwall S109 does not compromise the later conclusions regarding the largest clusters but merely suggests a similar failure mechanism due to working activities in this area.

\subsection{Frequency magnitude distributions and indications for different types of brittle failure}

As in former studies of mining seismicity (e.g. Richardson and Jordan, 2002; Finnie, 1999; Gibowicz and Kijko, 1994) a bimodal behaviour of the FMD is also observed when the whole catalogue is investigated (Fig. 10). This behaviour is generally explained by the superposition of two different seismic event classes consisting on the one hand of small magnitude events clustering in the immediate vicinity of the active mining and on the other hand of often stronger events linked to pre-existing geological discontinuities (Gibowicz and Kijko, 1994). Richardson and Jordan (2002) termed them type A and B events in the case of micro-seismicity recorded in South African gold mines and attributed the large number of small magnitude events (type A) to fracture dominated behaviour in the immediate vicinity of the active stope. The larger events constituting type B are explained by friction dominated behaviour on pre-existing geological structures. The identification of a particular event to one of the two types was achieved using a clustering algorithm utilizing the spatio-temporal event characteristics and a magnitude threshold. Finnie (1999) used a neural network approach to separate events from a South African gold mine into so-called "spurious" and "genuine" events corresponding to type A and B events, respectively. By this approach he was able to spatially separate these event types and correlate the "genuine" events with known tectonic structures. While type A or "spurious" events always occur in close proximity to the active mining, type B or "genuine" events can occur at some distance on tectonic structures. For the events in the present study the waveform-based cluster analysis is able to perform the separation into these different types as suggested by the FMDs and the spatio-temporal behaviour of the differently identified clusters.

The FMD observed for the whole HAMNET catalogue (Fig. 10) with a strong fall off above the completeness magnitude of about $M_{\mathrm{L}}=-0.4$ and a sharp inflection to a lower slope at about $M_{\mathrm{L}}=0.2$ resembles the observations from deep South African gold mines (Richardson and Jordan, 2002; Finnie, 1999). When analysing the FMDs of the largest identified clusters (Fig. 10) it is apparent that they exhibit clearly distinct characteristics. The largest clusters consist of small magnitude events, clustering within the confines of the longwall at the depth of active mining (Fig. 9) and closely correlated with the spatio-temporal migration of the facewall (Fig. 11). Thus these events seem to indicate the immediate energy release by fracturing of the rockmass directly adjacent to the mined out area and might be interpreted as type A or "genuine" events in the terminology of the former studies (Richardson and Jordan, 2002; Finnie, 1999). It is suggested that these events occur in a "low normal-stress environment" (Richardson and Jordan, 2002) and the high $b$ values observed for these events in the present study with values of up to about 2.5 are in agreement with such an interpretation. Several past studies showed that normal-stress environments are characterized by higher than normal $b$ values (e.g. Schorlemmer and Wiemer, 2005; Becker et al., 2010). The studies of the South African data sets also found high $b$ values for this event type reaching values of up to 3.6 (Finnie, 1999). The increase of $b$ value with time for this event type might be caused by changes in the temporally variable stress field around the mined out cavity or changes in the material parameters surrounding the mined out seams. Also the presence of older longwalls in the vicinity of the active one might 


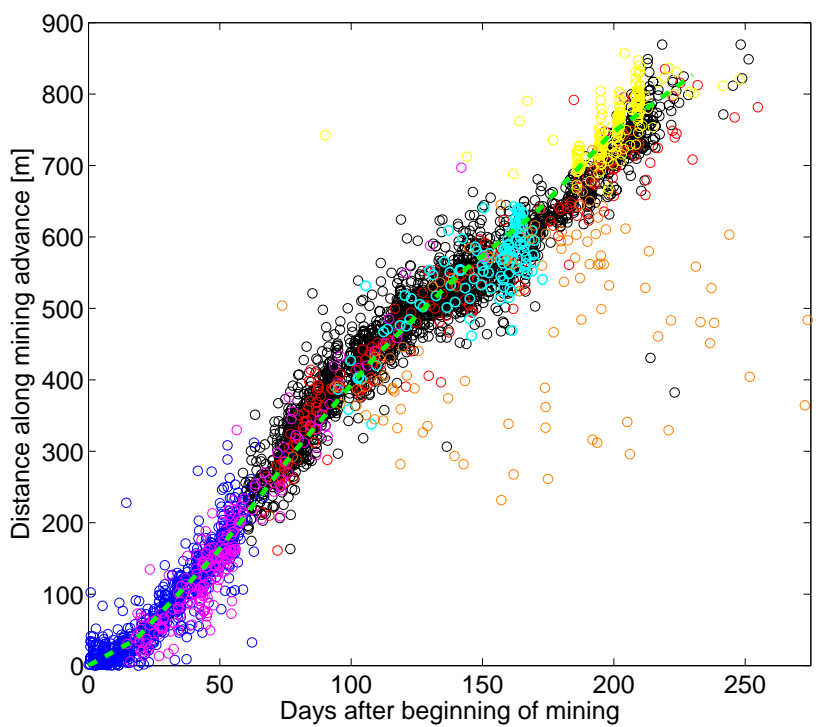

Fig. 11. Spatio-temporal migration of cluster events along the longwall. The broken green line indicates the spatio-temporal migration of the facewall position as reported by the mine operator.

contribute to these changes in the frequency-magnitude relation. Although the magnitude interval from which these $b$ values are calculated is rather narrow (from about $M_{\mathrm{L}}=$ -0.5 up to $M_{\mathrm{L}}=0.5$ to 1 ), the fit to the data is good (GFTtest values $>90)$ and the whole part above the completeness magnitude of the entire catalogue $\left(M_{\mathrm{L}}=-0.4\right)$ is linear. The results of the $b$ values are in agreement with the observation that during the beginning of mining the energy release in the vicinity of the mined out seam was considerably larger than during later times.

The FMDs of clusters containing larger magnitude events observed in this study generally do not follow a GR relation (Fig. 10). They exhibit an almost uniform magnitude distribution over the range $0 \leq M_{\mathrm{L}} \leq 1.2$. However, they also closely follow the progression of the facewall position as in Fig. 11 and are confined to the extent of the longwall (Fig. 9). Thus they are also indicative of a quick release of induced stresses in the immediate vicinity of the active facewall and seem to belong to the longwall face seismicity (LFS), as the two largest clusters. Whether there is a small temporal delay of these events with respect to the events of the largest clusters is hard to tell from Fig. 11, although there might be some indication for that. While such a delay would be a very interesting observation it will likely be small. This seems to suggest that these events are not type B events in the original sense of Richardson and Jordan (2002) but are more likely indicative of the failure of more competent layers as suggested for this and other coal mining areas (e.g. Bischoff et al., 2010a; Walter et al., 1997). The relative relocation of events belonging to these clusters suggests that their source depths are either slightly above or below the depth of active mining in agreement with the location of more competent

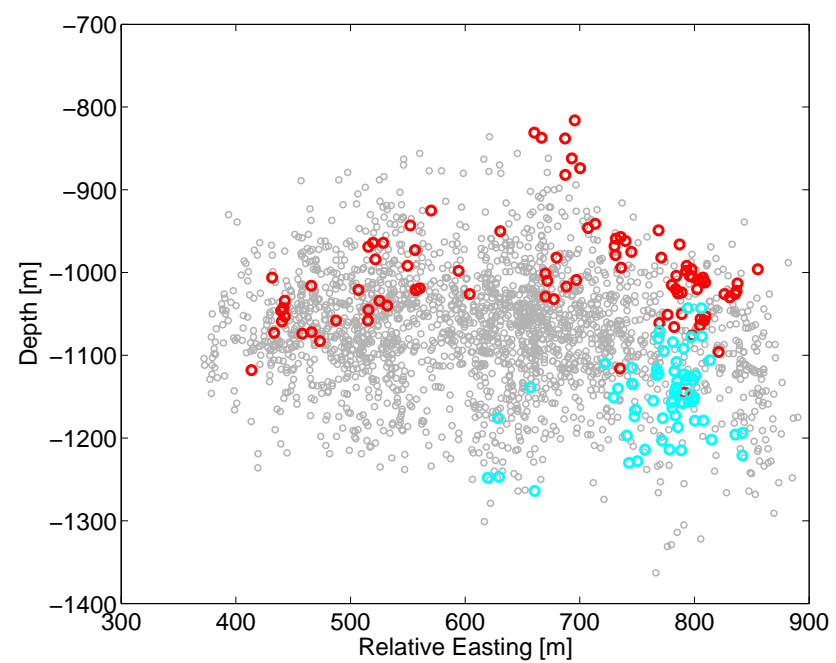

Fig. 12. Depth distribution of events that have been relocated with at least 20 cross-correlation times. Only the three largest clusters detected during the second half of mining operation within the confines of longwall S109 are depicted.

rock layers which exhibit violent failure behaviour in laboratory experiments conducted by Alber et al. (2009). The absence of such events in the South African data sets might be explained by the different geological settings. While there exists a succession of more or less competent sedimentary layers in the case of the Ruhr mining area, the situation in the much deeper South African gold mining environment is governed by much more homogeneous and harder rock strata.

The only cluster exhibiting considerable activity outside the borders of the longwall is the orange one in Fig. 9. Furthermore, this cluster also exhibits a FMD following the GR with a rather low $b$ value below 1 (Fig. 10) and a spatiotemporal event migration which is not in lockstep with the advance of the active facewall (Fig. 11). Seismic events comprising this cluster are thus candidates for activity on preexisting geological structures in correspondence to type B activity in Richardson and Jordan (2002).

Combining all the results from the cluster analysis, the relative event relocation and the evaluation of the FMDs of the largest clusters suggest that three different types of brittle failure dominating brittle seismic failure activity during the operation of longwall S109 are observed (Fig. 13). The suggested method of visual cluster extraction from sorted network SMs is able to identify these different failure processes without using any a priori assumptions. In general, additional stresses induced in the underground during the mining operation are quickly relieved by brittle failure as evidenced by the occurrence of microseismic activity in close proximity to the advancing facewall (Fig. 11 and Bischoff et al., 2010a). This stress release is either achieved by the majority of small magnitude events directly adjacent to the active facewall (I) or larger events occurring either slightly above 

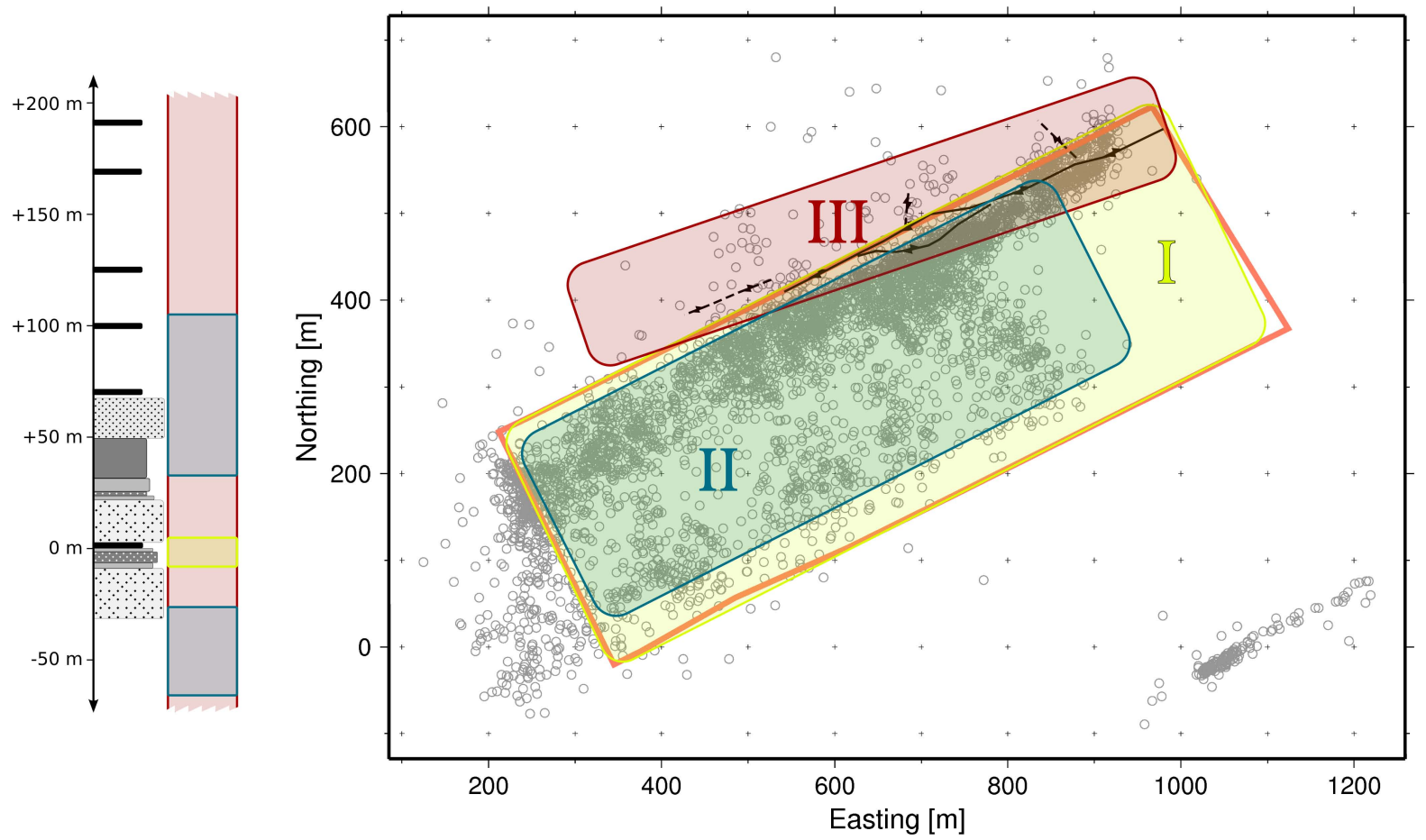

Legend:
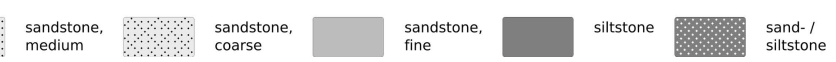

sand-I
siltstone

coal strike-slip fault

mapped (solid), extrapolated (dashed)

Fig. 13. Mapview of the relocated seismicity of the eight largest clusters (grey circles) and the three main types of brittle failure. On the left hand side there is a lithological column (modified after Alber et al. (2009)) illustrating the abundance of the observed failure types in the context of the geological setting underground. Events of type I (yellow) are directly accompanying the longwall face at mining level reflecting direct stress release by many small magnitude events. Type II (blue) are also events occurring horizontally within panel S109 but at shallower and greater depth reflecting potentially larger magnitude events in prominent rock layers which do not follow GR. Type III (dark red) are events which occur at all depths but concentrated at the northern edge of the longwall panel. This event type shows FMD following GR, thus this failure type has been associated to tectonic faults (information from personal conversation with the mine operator) which are concentrated in this area. Depth values are given relative to the mining level of panel S109. Although only a limited lithological profile is available the alternating sequences of more and less competent layers are also characteristic for the sedimentary bedding between the other indicated coal seams.

or below the active mining level (II). Furthermore, events at a larger distance to the facewall following a GR relation with low $b$ value are observed (III) (see Fig. 13). The fact that these different event classes partly overlap in their spatial and temporal extent as well as with respect to their magnitudes means that a separation of events based on some space-time clustering algorithm with an additional magnitude constraint would be challenging. However, due to their different waveform characteristics the separation into different clusters is the natural result of a cluster analysis without the necessity to a priori define the desired number of failure types as often necessary in simple neural network approaches. Moment tensor solutions as well as fault plane solutions calculated for the larger events of the three event types support the conclusion of distinct failure mechanisms for these three different event types (Sen et al., 2013; Fischer, 2010; Bischoff et al., 2010b). While events belonging to the same cluster show a high similarity with respect to strike, dip and rake, the differ- ent clusters exhibit considerable inter-cluster variability with respect to these parameters.

Figure 13 summarizes the results for the three different failure types in relation to longwall extent, event depth and lithology. The first type (I) is interpreted to be the seismic activity occurring in close proximity to the current coal extraction activity, possibly at only a few metres to tens of metres distance, representing brittle failure in rather incompetent material. Activity of the second type (II) is probably linked to failure in more competent layers at some vertical distance from the mined coal seam in competent rock layers within the alternating sedimentary bedding. These layers have the potential to accommodate more stress and thus may fail in larger events. The maximum magnitude of these events is most likely given by the geometric extent of the respective layers as shown in Bischoff et al. (2010a). The absence of a GR relation of their FMDs suggests that there is no fractal distribution of the possible rupture surfaces for this kind of 
events. They might only occur when the layers fail over their entire thickness. It is suggested that the competent layers accumulate stresses up to the strength of the layer itself resulting in events which only exhibit rather high magnitudes directly reflecting the thickness of the breaking sand-/siltstone layers (see also Alber et al., 2009; Bischoff et al., 2010a). Another explanation for the missing lower magnitude events in these event clusters could be that a minimum patch size with a critical slip distance is required in accordance with a slipweakening model of shear failure (e.g. Aki, 1987; Dieterich, 1986) as e.g. suggested by Richardson and Jordan (2002) for their type B events. This would require events belonging to the clusters to be pure shear failures within the more competent layers which is not in contradiction to results from moment tensor analysis (Sen et al., 2013). However, such a failure mechanism is generally associated with already preexisting fracture surfaces already exhibiting repeated seismic activity in the past.

Finally, the activity of the last type (III) might be linked to tectonic features partly outside the outline of the longwall (Fig. 11). Because the expected stress changes decrease quickly with increasing distance from the active longwall it is reasonable to assume that these events occur on structures already in critical state or close to failure. One candidate for such structures is a known steeply northward dipping fold of Variscan strike in the vicinity of the mining complex. Possible fault planes are the relatively weak interfaces between intercalated layers of sand-/siltstones, coarse sandstones and siltstones or steeply dipping faults at the bend of the fold. The fold in which the longwall is situated is dipping to the north at an angle of about $30^{\circ}$. Because these events exhibit Variscan striking and dipping angles (Sen et al., 2013; Fischer, 2010; Bischoff et al., 2010b) which are consistent with the known tectonic setting (see e.g. Behrmann et al., 1991; Brix et al., 1988; Drozdzewski, 1993) it is possible that these events represent the reactivation of older Variscan faults. However, these events are presumably induced and not triggered because these Variscan faults are not favourably oriented with respect to the recent natural stress field and thus no naturally occurring activity is expected on these older faults. This suggests that only the induced stress of the excavation is responsible for the seismic activity on these pre-existing zones of weakness. The observed FMD for this cluster with a $b$ value of 0.7 to 0.8 seems to support a possible tectonic influence for these events.

The present study shows that the different event types can be distinguished not only by their distinct frequency magnitude distributions and their locations but also by their waveforms, which means that a newly recorded event exhibiting high similarity to an already existing cluster can be immediately classified regardless of its location accuracy.

\section{Conclusions}

A new method for the identification of seismic event clusters based on waveform similarity is successfully tested on a large set of micro-seismic events recorded during the production phase of a coal longwall. The main advantage of this manual cluster identification method using sorted and visualized network SMs is the possibility to directly extract sub-clustering and interactively find clusters by visual inspection. The use of all available seismic event information, all three seismic traces of one station in the station matrix and all stations in the network matrix, makes the final result more robust. The influence of possible noise at one station is minimized and the averaging over all network stations partly compensates for the distance dependence of the waveform similarity. A comparison of the new sorting algorithm with the well known SL method using the same network matrix produced comparable results. However, in general, the visual extraction from the sorted SMs leads to larger distinct clusters when compared to the SL approach. Though the new method is more time consuming and needs the interaction of an analyst it results in an interpretable image of the seismicity pattern including the possible sub-clustering of events in one single processing step.

Cluster analysis with both methods results in the identification of several large clusters. Events belonging to these distinct clusters exhibit a clear spatial and temporal clustering. Utilizing the results from the relative relocation and an analysis of their FMDs they can be broadly divided into three different types possibly indicating different brittle failure mechanisms. This separation into different event types is the direct result of the waveform based clustering approach without any a priori assumptions. The first type, representing more than $50 \%$ of all events, occurs mainly at the depth of the active longwall mining, consists of low magnitude events in the range of $-1.5 \leq M_{\mathrm{L}} \leq 1.2$ and exhibits high $b$ values of around 2. Events of this type show a clear spatio-temporal migration in lockstep with the advancing longwall face indicating an instantaneous release of stresses in the vicinity of the longwall.

The second type consists of clusters which exhibit event locations on average slightly shallower or deeper than the mining operation level and FMDs not following GR. These events also show a clear spatio-temporal migration in lockstep with the advancing longwall face. It is suggested that these events represent brittle failure in competent finegrained sand-/siltstone layers and that these layers only feature brittle failure when the induced stresses overcome a value sufficient to break the whole thickness of these layers.

The third type is represented by a cluster of events located mainly to the north of the longwall panel in slightly shallower depths than the active mining. The $b$ value of this cluster is below 1 and in contrast to the previous clusters the events do not show a migration in lockstep with the active longwall face. The location of these events outside the confines of the 
longwall, the FMD of this activity as well as the presence of known faults in this area suggest the reactivation of preexisting tectonic structures.

Acknowledgements. We gratefully acknowledge the support of the mine personnel of Bergwerk Ost in Hamm and Michael Alber (RUB) for elucidating discussions on the layout of the mine, the geological and tectonic setting and the mining operation itself. This project was partly funded by the German Research Foundation (DFG) within the Collaborative Research Centre 526. The work of D. Becker for this project was funded by the DFG through grant BE4602/1-1. Most of the stations used here were provided by the GIPP-Pool at GFZ Potsdam (http://www.gfz-potsdam.de/portal/gfz/Struktur/Departments/ Department+2/sec22/infrastructure/GIPP).

Special Issue: "Rheology of the Earth - observations, laboratory experiments and numerical modelling from the micro- to the macro-scale" Edited by: H. Thybo and W. Friederich

\section{References}

Aki, K.: Maximum likelihood estimate of $\mathrm{b}$ in the formula $\log \mathrm{N}=$ a - bM and its confidence limit, B. Earthq. Res. I., 43, 237-239, 1965.

Aki, K.: Magnitude frequency relation for small earthquakes: a clue to the origin for $f_{\max }$ of large earthquakes, J. Geophys. Res., 89, 1349-1355, 1987.

Al Heib, M.: Numerical and Geophysical Tools Applied for the Prediction of Mine Induced Seismicity in French Coalmines, Int. J. Geosci., 3, 834-846, 2012.

Alber, M., Fritschen, R., Bischoff, M., and Meier, T.: Rock mechanical investigations of seismic events in a deep longwall coal mine, Int. J. Rock Mech. Min. Sci., 46, 408-420, 2009.

Amitrano, D.: Brittle-ductile transition and associated seismicity: Experimental and numerical studies and relationship with the $b$ value, J. Geophys. Res., 108, 2044, doi:10.1029/2001JB000680, 2003.

Aster, R. C. and Scott, J.: Comprehensive Characterization of Waveform Similarity in Microearthquake Data Sets, B. Seismol. Soc. Am., 83, 1307-1314, 1993.

Becker, D., Meier, T., Rische, M., Bohnhoff, M., and Harjes, H.-P.: Spatio-temporal microseismicity clustering in the Cretan region, Tectonophysics, 423, 3-16, 2006.

Becker, D., Cailleau, B., Dahm, T., Shapiro, S., and Kaiser, D.: Stress triggering and stress memory observed from acoustic emission records in a salt mine, Geophys. J. Int., 182, 933-948, 2010.

Behrmann, J., Drozdzewski, G., Heinrichs, T., Huch, M., Meyer, W., and Oncken, O.: Crustal-scale balanced cross sections through the Variscan fold belt, Germany: the central EGTsegment, Tectonophysics, 196, 1-21, 1991.

Bender, B.: Maximum likelihood estimation of b-values for magnitude grouped data, B. Seismol. Soc. Am., 73, 831-851, 1983.

Bischoff, M., Cete, A., Fritschen, R., and Meier, T.: Coal mining induced seismicity in the Ruhr Area, Germany, Pure Appl. Geophys., 167, 63-75, 2010a.
Bischoff, M., Fischer, L., Wehling-Benatelli, S., Fritschen, R., Meier, T., and Friederich, W.: Source mechanisms of mining induced seismic events in Hamm, in: Bochumer Geowissenschaftliche Arbeiten, edited by: Friederich, W., vol. 17, Sonderforschungsbereich 526, Institute for Geology, Mineralogy and Geophysics - Ruhr-University Bochum, 2010b.

Bischoff, M., Fischer, L., Wehling-Benatelli, S., Fritschen, R., Meier, T., and Friederich, W.: Spatio-temporal charcteristics of mining induced seismicity in the eastern Ruhr-area, in: Cahiers du Centre Européen de Géodynamique et de Séismologie, edited by: Ritter, J. and Oth, A., vol. 30, The European Center for Geodynamics and Seismology (ECGS), 2010c.

Brix, M. R., Drozdzewski, G., Greiling, R. O., Wolf, R., and Wrede, V.: The N Variscan margin of the Ruhr coal district (Western Germany): structural style of a buried thrust front?, Geol. Rundsch., 77, 115-126, 1988.

Deichmann, N. and Giardini, D.: Earthquakes Induced by the Stimulation of an Enhanced Geothermal System below Basel (Switzerland), Seismol. Res. Lett., 80, 784-798, 2009.

Dieterich, J. H.: A model for the nucleation of earthquake slip, in: Earthquake Source Mechanics, edited by: Das, S., Boatwright, J., and Scholz, C. H., Geophys. Monogr. Ser., 37, 37-47, AGU, 1986.

Douglas, A.: Joint hypocentre determination, Nature, 215, 47-48, 1967.

Drozdzewski, G.: The Ruhr coal basin (Germany): structural evolution of an autochtonous foreland basin, Int. J. Coal Geol., 23, 231-250, 1993.

Everitt, B.: Cluster Analysis, Arnold, 1993.

Finnie, G.: Using Neural Networks to Discriminate between Genuine and Spurious Seismic Events in Mines, Pure Appl. Geophys., 154, 41-56, 1999.

Fischer, K. D.: Seismological Observatory of the Ruhr-University Bochum Publications, http://www.gmg.ruhr-uni-bochum.de/ geophysik/seisobs/publications.html, 2013.

Fischer, L.: Herdmechanismen für bergbauinduzierte Ereignisse in Hamm mit Hilfe von HAMNET Daten, Master's thesis, RuhrUniversität Bochum, 2010.

Geller, R. J. and Mueller, C. S.: Four similar earthquakes in central California, Geophys. Res. Lett., 7, 821-824, 1980.

Gibowicz, S. J. and Kijko, A.: An Introduction to Mining Seismology, Academic Press, San Diego, 1994.

Gibowicz, S. J., Harjes, H.-P., and Schäfer, M.: Source parameters of seismic events at Heinrich Robert Mine, Ruhr Basin, B. Seismol. Soc. Am., 80, 88-109, 1990.

Got, J. L. and Fréchet, J.: Origins of amplitude variations in seismic doublets: source or attenuation process, Geophys. J. Int., 114 325-340, 1993.

Gutenberg, B. and Richter, C.: Frequency of earthquakes in California, B. Seismol. Soc. Am., 34, 185-188, 1944.

Hemmann, A., Meier, T., Jentzsch, G., and Ziegert, A.: Similarity of waveforms at stations Moxa and Plauen for the 1985/86 swarm, Stud. Geophys. Geod., 44, 602-607, 2000.

Köhler, N., Spies, T., and Dahm, T.: Seismicity patterns and variation of the frequency-magnitude distribution of microcracks in salt, Geophys. J. Int., 179, 489-499, 2009.

Kraft, T., Wassermann, J., and Igel, H.: High-precision relocation and focal mechanism of the 2002 rain-triggered earthquake 
swarms at Mt Hochstaufen, SE Germany, Geophys. J. Int., 167, 1513-1528, 2006.

Maurer, H. and Deichmann, N.: Microearthquake cluster detection based on waveform similarities, with an application to the western Swiss Alps, Geophys. J. Int., 123, 588-600, 1995.

Mintrop, L.: Die Erdbebenstation der Westfälischen Berggewerkschaftskasse in Bochum, Teil 1/2, Glückauf, 45, 357-366, 1909a.

Mintrop, L.: Die Erdbebenstation der Westfälischen Berggewerkschaftskasse in Bochum, Teil 2/2, Glückauf, 45, 393-403, 1909b.

Phillips, W.: Precise Microearthquake Locations and Fluid Flow in the Geothermal Reservoir at Soultz-sous-Forets, France, B. Seismol. Soc. Am., 90, 212-228, 2000.

Pujol, J.: Comments on the joint determination of hypocenters and station corrections, B. Seismol. Soc. Am., 78, 1179-1189, 1988.

Richardson, E. and Jordan, T. H.: Seismicity in Deep Gold Mines of South Africa: Implications for Tectonic Earthquakes, B. Seismol. Soc. Am., 92, 1766-1782, 2002.

Schorlemmer, D. and Wiemer, S.: Microseismicity data forecast rupture area, Nature, 434, 1086, 2005.
Sen, A. T., Cesca, S., Bischoff, M., Meier, T., and Dahm, T.: Automated Full Moment Tensor Inversion of Coal Mining Induced Seismicity, Geophys. J. Int., in press, 2013.

Waldhauser, F. and Ellsworth, W.: A Double Difference Earthquake Location Algorithm: Method and Application to the Northern Hayward Fault, B. Seismol. Soc. Am., 90, 1353-1368, 2000.

Walter, W. R., Heuze, F., and Dodge, D.: Seismic signals from underground cavity collapses and other mining related failures, in: Proc. of the 19th Annual Seismic Research Symposium on Monitoring a Comprehensive Test Ban Treaty, 23-25 September 1997, Orlando, Florida, 678-687, 1997.

Wiemer, S. and Schorlemmer, D.: ALM: An Asperity-based Likelihood Model for Carlifornia, Seismol. Res. Lett., 78, 134-140, 2007.

Wiemer, S. and Wyss, M.: Minimum Magnitude of Completeness in Earthquake Catalogs: Examples from Alaska, the Western United States, and Japan, B. Seismol. Soc. Am., 90, 859-869, 2000.

Wiemer, S. and Wyss, M.: Mapping spatial variability of the frequency-magnitude distribution of earthquakes, Adv. Geophys., 45, 259-302, 2002.

Wolfe, C.: On the mathematics of using difference operators to relocate earthquakes, B. Seismol. Soc. Am., 92, 2879-2892, 2002. 